\title{
Interactions between endothelial nitric oxide synthase and sex hormones in vascular protection in mice
}

\author{
Jeffrey B. Hodgin, Joshua W. Knowles, Hyung-Suk Kim, Oliver Smithies, \\ and Nobuyo Maeda
}

Department of Pathology and Laboratory Medicine, and Curriculum in Genetics and Molecular Biology, University of North Carolina at Chapel Hill, Chapel Hill, North Carolina, USA

Address correspondence to: Nobuyo Maeda, Department of Pathology and Laboratory Medicine, University of North Carolina at Chapel Hill, Chapel Hill, North Carolina 27599-7525.

Phone: (919) 966-6912; Fax: (919) 966-8800; E-mail: nobuyo@med.unc.edu.

Jeffrey B. Hodgin and Joshua W. Knowles contributed equally to this work.

Received for publication August 27, 2001, and accepted in revised form December 27, 2001.

\begin{abstract}
The vasculoprotective effects of sex hormones, particularly estrogens, have been attributed to their ability to increase the bioavailability of nitric oxide through activation of endothelial nitric oxide synthase (eNOS). To dissect the relative contribution in vivo of eNOS, sex hormones, and their interaction in two complex vascular phenotypes, hypertension and atherosclerosis, we used mice doubly deficient in eNOS and apoE (nnee) or lacking only apoE (NNee). Females and males were gonadectomized at 1 month of age and implanted either with control pellets or pellets releasing $17 \beta$-estradiol (E2). Hormonally intact nnee mice have elevated blood pressure (BP) and increased atherosclerosis compared with NNee mice, but on removal of gonads, BP and atherosclerosis decreased significantly in nnee mice but not in NNee mice. Three months of treatment with exogenous E2 dramatically reduced atherosclerosis and significantly lowered BP in both NNee and nnee mice compared with animals treated with control pellets. Thus exogenous E2 has strong BP-lowering and atheroprotective effects in apoE-deficient mice, but eNOS is not essential for either effect. Endogenous sex hormones, on the other hand, cause significant damage to the vasculature in the absence of eNOS, but these effects are overridden by interactions between eNOS and sex hormones.
\end{abstract}

J. Clin. Invest. 109:541-548 (2002). DOI:10.1172/JCI200214066.

\section{Introduction}

Men are generally at a greater risk for cardiovascular disease than are premenopausal women. After menopause, coronary heart disease rates and hypertension accelerate more quickly in women than in men of the same age, suggesting that sex hormones play a significant role in the development of cardiovascular disease $(1,2)$. Currently, however, the use of sex hormones as replacement therapy in humans is controversial. Epidemiological studies support a role for estrogen-replacement therapy, alone or in combination with a progestin, in the primary prevention of coronary heart disease in postmenopausal women, but randomized controlled studies have not shown a benefit for women with pre-existing disease (3). Although the use of exogenous androgens is associated with significant increases in risk of cardiovascular disease (4), a recent study suggests that natural androgens may correlate with lower coronary heart disease risk in men (5). In animal studies, $17 \beta$-estradiol (E2) protects against the development of atherosclerosis and hypertension (2, 6-10); likewise, testosterone has been shown to be atheroprotective in male mice and rabbits $(9,11,12)$.

The mechanism(s) for the protective effects of sex hormones are unclear. The modest ability of estrogenreplacement therapy to favorably alter the lipid profile accounts for only a limited part of the atheroprotection in humans (13), and in some animal studies E2 is fully protective without affecting plasma lipid levels (7-9). Additionally, the atheroprotective effects of androgens in mice result in large part from the local conversion of androgen to estradiol in the aorta by aromatase (12). Accordingly, attention has focused on local mediators that may contribute to the cardiovascular-protective effects of E2 through direct actions at the vessel wall.

Several lines of evidence have suggested that nitric oxide (NO) and E2 have related, protective effects on the vasculature. First, NO and E2 share many potentially atheroprotective functions, such as inhibiting leukocyte adhesion, vascular smooth muscle cell (VSMC) proliferation, and platelet aggregation, as well as causing vasodilation $(14,15)$ and blood pressure (BP) reduction $(16,17)$. Thus, absence of endothelial NO synthase (eNOS) in mice results in hypertension $(18,19)$, dysfunctional vasodilation $(20)$, and enhanced VSMC proliferation in response to injury (21). In apoEdeficient $\left(\right.$ Apoe $\left.^{-/-}\right)$mice, lack of eNOS results in increased atherosclerotic lesion size (22). Similarly, absence of E2 caused by gonadectomy contributes to dysfunctional vasodilation in monkeys (23), and enhances VSMC proliferation in response to injury in mice (14). Additionally, both eNOS gene expression and 
enzyme activity are affected by E2. Physiological levels of E2 stimulate eNOS mRNA and protein expression through long-term, receptor-dependent mechanisms $(24,25)$. Short-term exposure of cultured endothelial cells to E2 also activates eNOS activity without increasing the transcription of the eNOS gene (14). Third, inhibition of NO synthesis decreases some of the effects of $\mathrm{E} 2$ on the vasculature. For instance, treatment with the nonspecific NOS inhibitor L-NAME abolished the protective effect of $\mathrm{E} 2$ on vasodilator responsiveness and partly abrogated the antiatherogenic effect in cholesterol-clamped rabbits (25). However, in $\mathrm{Apoe}^{-/-}$mice, L-NAME treatment did not alter atheroprotection by E2 (26). It is important to note that a pharmacological approach is somewhat limited because L-NAME treatment affects all three NOS's, and the degree of inhibition of each enzyme is difficult to ascertain.

Here we describe our attempt to dissect the relative roles of sex hormones, eNOS, and their interactions in the cardiovascular system using genetically defined mouse models. Our data show that exogenous E2 reduces $\mathrm{BP}$ and atherosclerotic plaque size in gonadectomized mice lacking both eNOS and apoE (nnee mice) as effectively as it does in control mice lacking apoE alone (NNee mice), demonstrating that these effects are eNOS-independent. Yet we also find that gonadectomy in nnee mice abrogates the hypertension and increased atherosclerosis caused by the lack of eNOS in these mice, demonstrating an important interaction between endogenous hormones and eNOS.

\section{Methods}

Animals. The generation of mice doubly deficient for eNOS and apoE (nnee mice) from eNOS-deficient $\left(e \mathrm{NOS}^{-/-}\right)$mice (nn) (19) and Apoe ${ }^{-/-}$(ee) mice (27), both backcrossed at least six times to C57BL6/J mice, was described previously (22). At day 30, NNee and nnee mice were assigned into six study groups: gonadectomized and implanted with an E2-releasing pellet (see below for description of pellets) (NNee/E2 and nnee/E2, respectively), their respective gonadectomized control groups (NNee/Gdx and nnee/Gdx), and surgically intact NNee (NNee/I) and nnee (nnee/I) mice. Mice in the treatment group were anesthetized (using 100 $\mathrm{mg} / \mathrm{kg}$ ketamine and $8 \mathrm{mg} / \mathrm{kg}$ xylazine), gonadectomized, and implanted subcutaneously with either pellets designed to continuously release E2 at $6 \mu \mathrm{g} /$ day for 60 days (E2), or a control pellet without E2 (Gdx) (Innovative Research of America, Sarasota, Florida, USA). Pellets were replaced 60 days after surgery, and the mice were sacrificed 30 days later (at 4 months of age), after a 2- to 4-hour fast. Both males and females were studied. Mice were maintained in specific pathogen-free conditions on standard mouse chow (Prolab RMH 3000; PMI Nutrition International, Brentwood, Missouri, USA). Mouse experiments were carried out under protocols approved by the Institutional Animal Care and Use Committee of the University of North Carolina at Chapel Hill.
Plasma lipid analysis. Total cholesterol, HDL, and triglycerides were measured colorimetrically using commercially available kits (Sigma-Aldrich, St. Louis, Missouri, USA) in plasma collected at sacrifice after a 4-hour fast (27). Plasma samples were stored at $-20^{\circ} \mathrm{C}$ until they were assayed.

Systolic BP analysis. BP was measured noninvasively using a tail-cuff method on conscious, restrained mice as described (28), when the animals were between 3 months and 4 months of age. BP measured by this method correlates well with intra-arterial measurements in mice $(28,29)$. All BPs were calculated as the average of 5-10 measurements per day for 6 consecutive days.

Atherosclerotic lesion analysis. Animals were sacrificed at 4 months of age, and the vascular tree was perfused with $4 \%$ paraformaldehyde under physiological pressure. Segments of the aortic sinus were embedded, sectioned, and stained as described previously (27); four sections, chosen by strict anatomical criteria, were used for morphological evaluation. The average lesion size in the four sections was taken to represent the lesion size of each animal.

RNA extraction and RT-PCR. Kidneys and livers were snap-frozen in liquid nitrogen and stored at $-80^{\circ} \mathrm{C}$ in RNAlater solution (Ambion Inc., Austin, Texas, USA). RNA samples were prepared from homogenized tissue using an ABI PRISM 6700 Automated Nucleic Acid Workstation (PE Biosystems, Foster City, California, USA). Kidney renin, kidney angiotensin II receptor type $\mathrm{I}\left(\mathrm{AT}_{1 \mathrm{~A}}\right.$ receptor), and liver angiotensinogen $\mathrm{mRNA}$ levels were determined by real-time quantitative RT-PCR with the ABI PRISM 7700 Sequence Detection System. Primers for renin were $5^{\prime}$-ACAGTATCCCAACAGGAGAGACAAG-3' and 5'-GCACCCAGGACCCAGACA-3'; the probe for renin detection was $5^{\prime}$-6-FAM-TGGCTCTCCATGCCATGGACATCC-TAMRA-3'. Primers for $\mathrm{AT}_{1 \mathrm{~A}}$ receptor were $5^{\prime}$-CACATTCTTGGATGTGCTGA- $3^{\prime}$ and $5^{\prime}$ AGAGGGTTCAGGCAGTTGTT-3'; the probe for $\mathrm{AT}_{1 \mathrm{~A}}$ receptor detection was 5'-6-FAM-TGCCATGCCCATAACCATCTGCA-TAMRA- $3^{\prime}$. Primers for angiotensinogen were $5^{\prime}$-GATGAGAGGTTTCTCTCAGC- $3^{\prime}$ and $5^{\prime}$-TTGCCAGTGCCGGAGATCAT- $3^{\prime}$; the probe for angiotensinogen detection was 5'-6-FAM-ACACCGAGATGCTGTTGTCCACCCA-TAMRA-3'. All reactions included $\beta$-actin amplification as an internal standard, with primers $5^{\prime}$-CTGCCTGACGGCCAAGTC-3' and $5^{\prime}$-CAAGAAGGAAGGCTGGAAAAGA- $3^{\prime}$. The probe for $\beta$-actin detection was $5^{\prime}$-TET-CACTATTGGCAACGAGCGGTTCCG-TAMRA-3'. TET, FAM, and TAMRA probe tags were purchased from PE Biosystems.

Statistical analysis. Statistical analyses were performed with JMP software, version 3.2.6 (SAS Institute Inc., Cary, North Carolina, USA). Data were first analyzed for sex-related effects using factorial ANOVA. There were no sex-related differences in BP $(P=0.20)$, as we have shown previously, and thus the data from male and female mice were combined $(19,22,29)$. There was a strong sex-related effect in atherosclerotic lesion size $(P<0.001)$, so this data was analyzed separately. For 


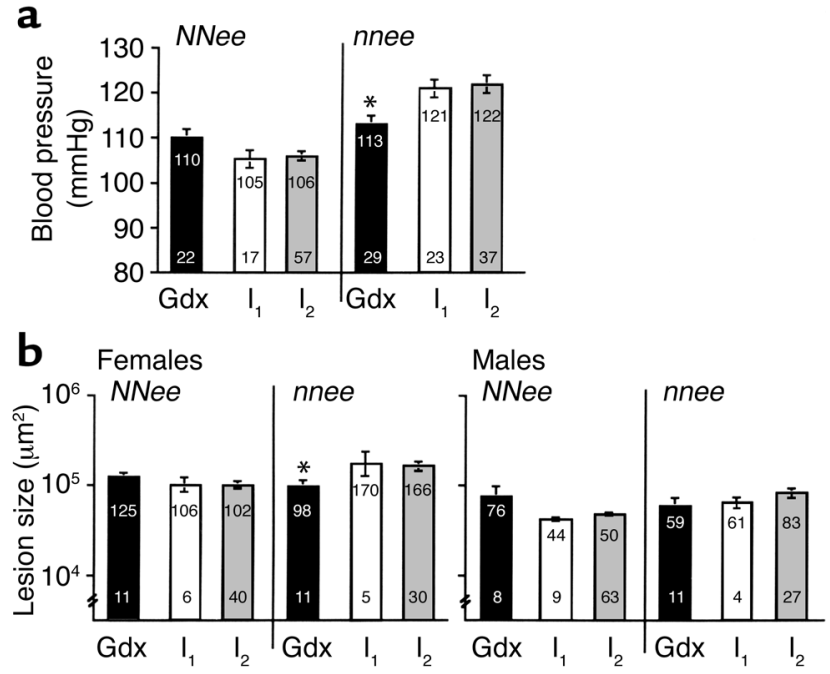

effect of gonadectomy, two-way ANOVA was used, and surgical status and genotype were used as the two grouping variables. Genotype and E2 status were used as the two grouping variables for effects of E2. Further analysis to determine the significance of main effects and/or interactions used the Bonferroni multiple comparison procedure (for $\mathrm{BP}$ and lesion analysis) or the Tukey-Kramer honestly significant difference test (for gene expression data) to correct type I errors. Statistical significance was as set at 0.05 for all comparisons. The mean values of BP and atherosclerotic lesion size of surgically intact NNee and nnee mice analyzed in the current study were not different from the mean values of cumulative data from surgically intact NNee and nnee mice accumulated during the 3-year period that includes the experiments we are currently reporting. During this period, there were no changes in our animal husbandry or in personnel who measured the BP and atherosclerosis of these mice. Analysis showed that there have been no systematic alterations in the mean of either BP or atherosclerotic lesion size between sets of data during this period. Periodic addition of new data did not alter the cumulative mean significantly, but reduced the SEM as expected $(r=0.98-0.99)$. Therefore, we also used cumulative data from surgically

\section{Figure 1}

$\mathrm{BP}$ and atherosclerotic lesion size in NNee and nnee mice, either gonadectomized and implanted with a control pellet ( $G d x)$ or surgically intact (I). $I_{1}$, current data; $I_{2}$, cumulative data. The lower number in each bar is sample size $(n)$. The upper number in each bar is mean $\mathrm{BP}(\mathrm{mmHg})$ or lesion size $\left(\times 10^{3} \mu \mathrm{m}^{2}\right)$, with SEM represented as a vertical line. (a) The BP measurements for male mice (10 Gdx NNee and $14 \mathrm{Gdx}$ nnee; $35 \mathrm{I}_{2}$ NNee and $19 \mathrm{I}_{2}$ nnee) and female mice (12 Gdx NNee and $15 \mathrm{Gdx}$ nnee; $22 \mathrm{I}_{2}$ NNee and $18 \mathrm{I}_{2}$ nnee; see Results for number of $I_{1}$ male and female mice) were combined as described in Methods. Removal of endogenous sex hormones by gonadectomy results in a significant reduction in $\mathrm{BP}$ in nnee mice $\left({ }^{*} P<0.05\right.$ versus $\mathrm{I}_{2}$, Student's $t$ test with Bonferroni correction for multiple comparisons). (b) Removal of endogenous sex hormones by gonadectomy results in a significant decrease in atherosclerotic lesion size in nnee mice $\left({ }^{*} P<0.05\right.$ versus $I_{2}$, Student $t$ test with Bonferroni correction for multiple comparisons).

intact animals, some of which were included in our previous publication (22), in our analysis of intact versus gonadectomized, control-treated animals.

\section{Results}

Effects of gonadectomy in NNee and nnee mice. Endogenous production of sex hormones in female and male apoE-deficient mice that were wild-type or deficient in eNOS (NNee or nnee mice) was terminated at 1 month of age by gonadectomy, and was replaced by subcutaneously implanted E2-releasing pellets or with control pellets without hormone for 3 months. In what follows, NNee/E2 and nnee/E2 respectively indicate gonadectomized NNee and gonadectomized nnee mice with E2-releasing pellets; NNee/Gdx and nnee/Gdx indicate gonadectomized NNee and nnee mice with pellets lacking hormone. NNee/I and nnee/I indicate hormonally intact mice.

In the present study, we found that surgically intact (and therefore hormonally intact) nnee mice (nnee/I mice) are hypertensive compared with $N$ Nee/I mice ( $\mathrm{BP}=121 \pm 2 \mathrm{mmHg}, n=12$ males and 11 females, versus $105 \pm 2 \mathrm{mmHg}, n=10$ males and 7 females, respectively) and have increased atherosclerosis $\left(170 \times 10^{3} \pm\right.$ $41 \times 10^{3} \mu \mathrm{m}^{2}, n=5$, versus $106 \times 10^{3} \pm 25 \times 10^{3} \mu \mathrm{m}^{2}$,

Table 1

Body weight, uterine weight, and total cholesterol in gonadectomized NNee and nnee mice

\begin{tabular}{|c|c|c|c|c|c|c|}
\hline Study group & Sex & $n$ & $\begin{array}{l}\text { Body wt } \\
\text { at gonadectomy }\end{array}$ & $\begin{array}{c}\text { Body wt } \\
\text { at euthanasia }(\mathrm{g})\end{array}$ & $\begin{array}{c}\text { Uterine } w t \\
(\mathrm{mg} / \mathrm{g} \text { body } w \mathrm{t})\end{array}$ & $\begin{array}{c}\text { Total cholesterol } \\
(\mathrm{mg} / \mathrm{dl})\end{array}$ \\
\hline \multirow[t]{2}{*}{ NNee/Gdx } & $\mathrm{F}$ & 12 & $15.3 \pm 0.5$ & $22.0 \pm 0.5$ & $0.67 \pm 0.2$ & $402 \pm 21$ \\
\hline & M & 8 & $18.3 \pm 0.5$ & $21.8 \pm 0.8$ & - & $450 \pm 31$ \\
\hline \multirow[t]{2}{*}{ NNee/E2 } & $\mathrm{F}$ & 10 & $15.3 \pm 0.7$ & $21.7 \pm 0.6$ & $6.3 \pm 0.4^{\mathrm{A}}$ & $275 \pm 20^{A}$ \\
\hline & M & 10 & $19.1 \pm 0.8$ & $24.7 \pm 0.7$ & - & $309 \pm 29^{A}$ \\
\hline \multirow[t]{2}{*}{ nnee/Gdx } & $\mathrm{F}$ & 11 & $14.6 \pm 0.6$ & $20.2 \pm 0.7$ & $0.63 \pm 0.15$ & $438 \pm 18$ \\
\hline & M & 11 & $15.5 \pm 0.9^{B}$ & $18.8 \pm 1.0^{\mathrm{B}}$ & - & $503 \pm 36$ \\
\hline \multirow[t]{2}{*}{ nnee/E2 } & $\mathrm{F}$ & 9 & $15.6 \pm 0.6$ & $20.5 \pm 0.6$ & $5.70 \pm 0.17^{\mathrm{A}}$ & $284 \pm 30^{\mathrm{A}}$ \\
\hline & M & 7 & $16.2 \pm 0.9^{B}$ & $21.2 \pm 1.0^{B}$ & - & $279 \pm 41^{A}$ \\
\hline
\end{tabular}

Study group includes gonadectomized animals implanted with control or E2-releasing pellets. Data is presented as mean $\pm \mathrm{SEM}$. ${ }^{\mathrm{A} P}<0.01$ versus control-treated group of same genotype. ${ }^{B} P<0.05$ by two-way ANOVA for effect of genotype versus NNee. 


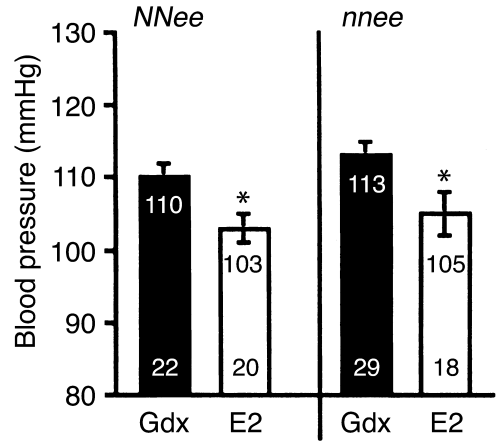

\section{Figure 2}

$\mathrm{BP}$ in gonadectomized NNee and nnee mice treated with a control pellet $(\mathrm{Gdx})$ or an E2-containing pellet. Lower number in each bar is sample size $(n)$. Upper number in each bar is mean BP, with SEM represented as a vertical line. The BP measurements for male (10 NNee and 14 nnee Gdx, and 10 NNee and 9 nnee E2) and female (12 NNee and 15 nnee Gdx; 10 NNee and 9 nnee E2) mice were combined as described in Methods. In NNee and nnee mice, exogenous E2 treatment results in a significant decrease in BP. ${ }^{*} P<0.05$ versus Gdx mice of the same genotype (Student $t$ test with Bonferroni correction for multiple comparisons).

$n=6$, for females; $61 \times 10^{3} \pm 30 \times 10^{3} \mu \mathrm{m}^{2}, n=4$, versus $44 \times 10^{3} \pm 6 \times 10^{3} \mu \mathrm{m}^{2}, n=9$, for males, respectively) (Figure 1, white bars). These observations are in complete agreement with previous findings by our group (22) and others (30). However, we were surprised to find that gonadectomy in nnee mice given control pellets (nnee/Gdx) appeared to reduce their BP and lesion sizes to the extent that they no longer differed from NNee/Gdx mice (Figure 1, black bars). Analysis by two-way ANOVA revealed a statistically significant interaction between genotype and gonadectomy for $\mathrm{BP}(P<0.02)$ for both sexes and for lesion size in females $(P<0.05)$, but not for lesion size in males $(P=0.26)$. Pairwise comparisons (with the Bonferroni correction) did not demonstrate significance because of the small number of hormonally intact NNee/I and nnee/I mice in this study.

However, because we have been analyzing these animals for some time, a better estimate of the BP and lesion measurements for NNee/I and nnee/I mice can be obtained from the data we have accumulated during the past 3 years. No systematic alterations in the mean values of either BP or atherosclerosis were present during this period (see Methods). When we used the cumulative data for hormonally intact nnee mice for comparison, gonadectomy at 1 month of age significantly reduced $\mathrm{BP}$ in nnee mice (by $9 \mathrm{mmHg}, P<0.05$ ) (Figure 1a, black bars versus gray bars). In contrast, the mean $\mathrm{BP}$ of gonadectomized NNee mice was higher than that of hormonally intact NNee mice by $4 \mathrm{mmHg}$, although the difference was not significant $(P=0.34)$. Gonadectomy also resulted in a $40 \%$ decrease in lesion size in female nnee mice $(P<0.05)$ and a 30\% (but nonsignificant) decrease in lesion size in male nnee mice. Together, these data establish a highly significant interaction between genotype and gonadectomy in both sexes $(P<0.005$ for BP and $P<0.01$ for lesion size). Since gonadectomy slightly increases both BP and lesion size in NNee mice, but significantly reduces them in nnee mice, these results demonstrate that the hypertensive and atherogenic effect of an absence of eNOS in $A p o e^{-/-}$mice is dependent on the presence of endogenous sex hormones.

Efficacy of E2 treatment. Exogenous E2 treatment achieved the desired hormonal status in the experimental animals (Table 1). Pellets that continuously release $6 \mu \mathrm{g}$ of E2 per day and lead to plasma E2 levels within a physiological range $(70-100 \mathrm{pg} / \mathrm{ml})$ were used based on our previous work, which demonstrated that this dosage reproducibly lowers plasma lipids and plaque size in NNee mice (31). E2 administration increased uterine weight to almost tenfold that of gonadectomized control-treated females of either genotype $(6.3 \pm 0.4 \mathrm{mg} / \mathrm{g}$ body wt versus $0.67 \pm 0.2$ $\mathrm{mg} / \mathrm{g}$ body wt for NNee/E2 and nnee/Gdx females, respectively; and $5.7 \pm 0.2$ versus $0.63 \pm 0.2$ for nnee/E2 and nnee/Gdx females, respectively; $P<0.01$ ). Mice given E2 had a mean uterine weight that was twice that of surgically intact NNee/I and nnee/I females (3.1 \pm 0.4 $\mathrm{mg} / \mathrm{g}$ body wt and $2.7 \pm 0.3 \mathrm{mg} / \mathrm{g}$ body wt, respectively; $P<0.05)$. The effect of E2 treatment on uterine weight was highly significant $(P<0.001$, two-way ANOVA). Genotype had no effect $(P=0.30)$ on uterine weight, nor was there any interaction between genotype and treatment $(P=0.37)$. In addition, E2 treatment had no effect on body weight. However, both male and female nnee mice weighed less than NNee mice $(P<0.05)$, in agreement with previous reports (19). As expected, E2 treatment reduced total plasma cholesterol by approximately $30 \%$ in all groups compared with control-treated animals $(P<0.01)$. The effect of E2 treatment on total plasma cholesterol was highly significant for both sexes $(P<0.001)$, but the effect of genotype $(P=0.32)$ and the interaction between treatment and genotype $(P=0.50)$ were not significant.
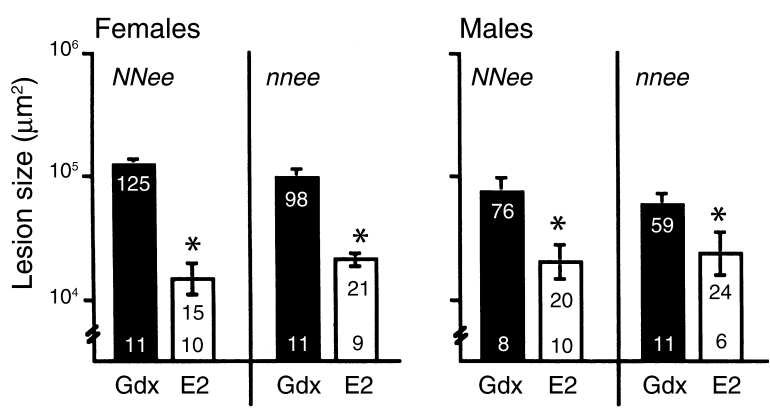

\section{Figure 3}

Atherosclerotic lesion size in gonadectomized NNee and nnee mice treated with a control pellet or an E2-containing pellet. Lower number in each bar is sample size $(n)$. Upper number in each bar is mean lesion size $\left(\times 10^{3} \mu \mathrm{m}^{2}\right)$, with SEM represented as a vertical line. In male and female mice of both genotypes, exogenous E2 treatment significantly reduces atherosclerotic lesion size. ${ }^{*} P<0.05$ versus $\mathrm{Gdx}$ mice of the same sex and genotype (Student $t$ test with Bonferroni correction for multiple comparisons). 
Table 2

Effects of E2 treatment and gonadectomy on the RAS

mRNA (pg/ $\mu \mathrm{g}$ total RNA)

\begin{tabular}{lccrc} 
Study group & Sex & \multicolumn{1}{c}{ Renin $(n)$} & $\mathrm{AT}_{1 \mathrm{~A}}$ receptor $(n)$ & Angiotensinogen ( $n)$ \\
NNee/I & $\mathrm{F}$ & $134 \pm 15(5)$ & $10.1 \pm 0.4(5)$ & $473 \pm 30(4)$ \\
& $\mathrm{M}$ & $58 \pm 9(6)^{\mathrm{A}}$ & $9.5 \pm 0.9(6)$ & $484 \pm 78(5)$ \\
NNee/Gdx & $\mathrm{F}$ & $135 \pm 4(5)$ & $9.3 \pm 0.4(5)$ & $484 \pm 56(4)$ \\
& $\mathrm{M}$ & $49 \pm 4(4)^{\mathrm{A}}$ & $8.8 \pm 0.7(4)$ & $485 \pm 11(4)$ \\
NNee/E2 & $\mathrm{F}$ & $134 \pm 8(3)$ & $9.7 \pm 0.6(3)$ & $435 \pm 19(4)$ \\
& $\mathrm{M}$ & $62 \pm 19(5)^{\mathrm{A}}$ & $7.4 \pm 0.9(5)$ & $516 \pm 46(5)$ \\
nnee/I & $\mathrm{F}$ & $63 \pm 8(5)^{\mathrm{B}}$ & $9.1 \pm 0.4(5)$ & $560 \pm 64(5)$ \\
& $\mathrm{M}$ & $49 \pm 15(5)$ & $7.1 \pm 0.9(5)$ & $441 \pm 29(5)$ \\
nnee/Gdx & $\mathrm{F}$ & $90 \pm 14(5)$ & $9.7 \pm 0.7(5)$ & $532 \pm 34(5)$ \\
& $\mathrm{M}$ & $67 \pm 8(7)$ & $10.9 \pm 2.2(5)$ & $503 \pm 80(7)$ \\
nnee/E2 & $\mathrm{F}$ & $129 \pm 10(5)$ & $7.9 \pm 0.7(5)$ & $495 \pm 53(6)$ \\
& $\mathrm{M}$ & $75 \pm 21(4)$ & $7.8 \pm 1.5(4)$ & $531 \pm 12(2)$
\end{tabular}

Study groups include gonadectomized mice implanted with either control pellets (Gdx) or E2-containing pellets, and surgically intact (I) mice. ${ }^{A} P<0.05$ versus females of the same genotype/treatment group. Data represent mean mRNA amount ( $\mathrm{pg} / \mathrm{\mu g}$ total $\mathrm{RNA}) \pm \mathrm{SEM}$. ${ }^{\mathrm{B} P}<0.05$ versus all other females except nnee/Gdx.

BP in E2-treated versus control-treated NNee and nnee mice. To determine the relationship between eNOS, exogenous $\mathrm{E} 2$, and $\mathrm{BP}$ regulation, we measured the BP of gonadectomized control-treated and E2-treated NNee and nnee mice (Figure 2). Compared with gonadectomized control-treated animals, both NNee/E2 and nnee/E2 animals had a modest but significant reduction in $\mathrm{BP}$ as a result of the $\mathrm{E} 2$ treatment $(7 \mathrm{mmHg}$ and $8 \mathrm{mmHg}$, respectively, $P<0.05$ by Student's $t$ test with Bonferroni correction). The effect of E2 treatment was highly significant by two-factorial ANOVA $(P<0.001)$. The genotype of the mice also had a significant effect; the nnee mice had slightly higher BPs than the NNee mice, regardless of treatment $(P<0.05)$, but there was no interaction between genotype and treatment $(P=0.78)$. Thus, the reduction in BP induced by exogenous E2 treatment in apoE-deficient mice is independent of the presence or absence of eNOS.

Atherosclerotic lesion size in E2-treated versus control-treated NNee and nnee mice. To determine the role of eNOS in the inhibition of atherosclerotic progression by exogenous E2, atherosclerotic lesion size was measured in the proximal aorta in gonadectomized control-treated and E2-treated NNee and nnee mice of both sexes. Mean lesion sizes in females and males were analyzed separately because surgically and hormonally intact $A p o e^{-/-}$ males have lesion sizes that are about half that of the females (32). Exogenous E2 was highly effective in inhibiting atherosclerotic lesion progression in all treatment groups, regardless of sex or genotype (Figure $3)$. Both NNee/E2 and nnee/E2 females had dramatic reductions in lesion size ( $88 \%$ and $78 \%$, respectively) compared with gonadectomized control-treated NNee/Gdx and nnee/Gdx females $(P<0.001)$. Similarly, compared with control-treated NNee/Gdx and nnee/Gdx males, the reduction in lesion size caused by E2 treatment of NNee and nnee males was also signifi- cant $(74 \%$ and $59 \%$, respectively; $P<0.03)$. The effect of E2 treatment was highly significant for both sexes $(P<0.001)$, but the effect of genotype and the interaction between E2 treatment and genotype were not $(P>0.20)$. These data demonstrate that most of the atheroprotective effect of exogenous E2 on lesion size is independent of the presence or absence of eNOS in both female and male mice.

Effects of gonadectomy and exogenous E2 treatment on the renin-angiotensin system. Because the reninangiotensin system (RAS) plays a major role in BP homeostasis, changes in the expression of the genes in the RAS could account for the modulation of BP and lesion size in NNee and nnee mice by gonadectomy and exogenous E2 treatment. We measured kidney renin, kidney $\mathrm{AT}_{1 \mathrm{~A}}$ receptor, and liver angiotensinogen mRNA from animals in each study group using RT-PCR to quantitate gene expression (Table 2). There were no significant differences in kidney $\mathrm{AT}_{1 \mathrm{~A}}$ receptor or liver angiotensinogen mRNA levels between any of the study groups. The male NNee mice, regardless of treatment or surgical status, expressed renin in the kidney at approximately half the level found in females $(P<0.05)$, and the female nnee mice had lower renin levels than female NNee mice had $(P<0.05$, effect of genotype). Thus the data suggest that the sex-related difference in renin expression is either independent of gonadal hormones or has been determined by the time of gonadectomy. In addition, we observed a trend toward an increase in renin levels in both female and male nnee mice in which BP was reduced by gonadectomy or by E2 treatment. Because gonadectomy and E2 treatment both reduce $\mathrm{BP}$ in nnee mice, the marginal increase in renin expression is more likely to be a consequence of a homeostatic response to reduced BP than a result of the differences in the hormonal status of the animal. Taken together, these results indicate that changes in expression of genes of the RAS do not play a significant role in the BP-reducing or the antiatherogenic effects of exogenous E2 treatment, or the effects of gonadectomy in nnee mice.

\section{Discussion}

The studies described here demonstrate that the hypertensive and atherogenic effects of an absence of eNOS in $A p o e^{-/-}$mice depends upon the presence of endogenous sex hormones, as evidenced by the reduction of BP and atherosclerosis by gonadectomy in nnee mice. In contrast, gonadectomy increased lesion size and tended to raise BP in NNee mice. Our study also found that continuous treatment with exogenous E2 reduces $\mathrm{BP}$ and atherosclerosis equally in gonadectomized nnee and NNee mice, demonstrating that eNOS is not essential for the vasculoprotective function of exogenous E2. The finding that atheroprotection by exogenous E2 in Apoe ${ }^{-/-}$mice is independent of 
eNOS confirms and extends the earlier study by Elhage et al. that showed that pharmacological inhibition of NO production did not influence the reduction of atherosclerosis by exogenous $\mathrm{E} 2$ in $A p o e^{-/-}$mice (26). The eNOS-independent lowering of BP by E2 that we observed here has not been described previously. This BP-lowering effect of E2 was not accompanied by changes in gene expression of kidney $\mathrm{AT}_{1 \mathrm{~A}}$ receptor, kidney renin, or liver angiotensinogen.

The finding that gonadectomy affects both BP and atherosclerosis differently in NNee and nnee mice was unexpected. The reduction of $\mathrm{BP}$ and lesion size caused by gonadectomy in nnee mice suggests that the hypertensive and atherogenic effects of eNOS deficiency are largely dependent on endogenous sex hormones. This implies that sex hormones have contradictory effects with respect to vascular action, regulating some target genes or pathways that protect and others that damage the vasculature. For example, in addition to upregulating eNOS, E2 induces VEGF in VSMCs (33), which may then contribute to plaque progression (34). Similarly, BP may be influenced by sex hormones through activation of the local RAS in the vasculature (35).

The vascular functions of the sex hormones and of eNOS are so intimately intertwined in vivo that the relative roles of the individual components are difficult to dissect. The path diagram in Figure 4 is our attempt to represent the contribution of each component and their interactions with respect to two vascular phenotypes, BP and atherosclerosis, as revealed by our current studies of genetically altered mice. In Figure 4, left panel, the differences between the mean BPs of six groups of Apoe ${ }^{-/-}$ mice that have been measured in this study (in rectangles and placed on the vertical scale) are used as an index (the black arrows indicate statistically significant differences and the gray arrows show nonsignificant differences). The effects of the presence of different components (in black boxes) are calculated from the differences between relevant groups. For example, nnee/I differs from nnee/Gdx by the presence and function of gonad-derived hormone $(\mathrm{H})$ in the absence of eNOS. We find that $\mathrm{H}$, deduced from the BP difference between nnee/I and nnee/Gdx mice, has a significant effect on BP, raising it by $9 \mathrm{mmHg}$. Similarly, NNee/Gdx mice differ from nnee/Gdx mice by the function of eNOS $(\mathrm{N})$ in the absence of sex hormones. Likewise, NNee/I mice differ from nnee/I mice by the presence of $\mathrm{N}$ and of $\mathrm{H} \bullet \mathrm{N}$ (which represents the interactions between hormones and eNOS). In the absence of hormones, eNOS has only a small, nonsignificant lowering effect on $\mathrm{BP}(-3 \mathrm{mmHg})$, but in the presence of the hormones, eNOS has a large and significant BP-lowering contribution $(-13 \mathrm{mmHg} ; \mathrm{H} \bullet \mathrm{N})$. The net consequence is that hormonally intact NNee/I mice have only slightly lower $\mathrm{BP}(-4 \mathrm{mmHg})$ than the $\mathrm{NNee} / \mathrm{Gdx}$ mice have, because the BP-lowering effect of $\mathrm{H} \bullet \mathrm{N}(-13 \mathrm{mmHg})$ overrides the BP-raising effect of $\mathrm{H}$ $(+9 \mathrm{mmHg})$; whereas NNee/I mice have significantly lower $\mathrm{BP}(-16 \mathrm{mmHg})$ than have nnee/I mice, which lack both $\mathrm{H} \bullet \mathrm{N}(-13 \mathrm{mmHg})$ and $\mathrm{N}(-3 \mathrm{mmHg})$ effects. Similar comparisons reveal that $\mathrm{E} 2$ in the absence of eNOS (E2 in Figure 4) lowers BP significantly (-8 $\mathrm{mmHg}$ ), whereas the interactive effect between eNOS and $\mathrm{E} 2(\mathrm{~N} \bullet \mathrm{E} 2)$ is negligible.

The relative contributions of each component in atherosclerosis are strikingly similar to their effects on BP, as illustrated in the right panel of Figure 4, which uses the differences in plaque size at the aortic root as an index. Because the effects in males and females were generally the same in both relative amount and direction, the averages of the mean plaque sizes in males and females were used to calculate the effects of each component. As with BP, there is a significant atheropromotive (accelerating) effect provided by $\mathrm{H}\left(+46 \times 10^{3} \mu \mathrm{m}^{2}\right)$, and strong atheroprotective (decelerating) effects by
$\mathrm{BP}$

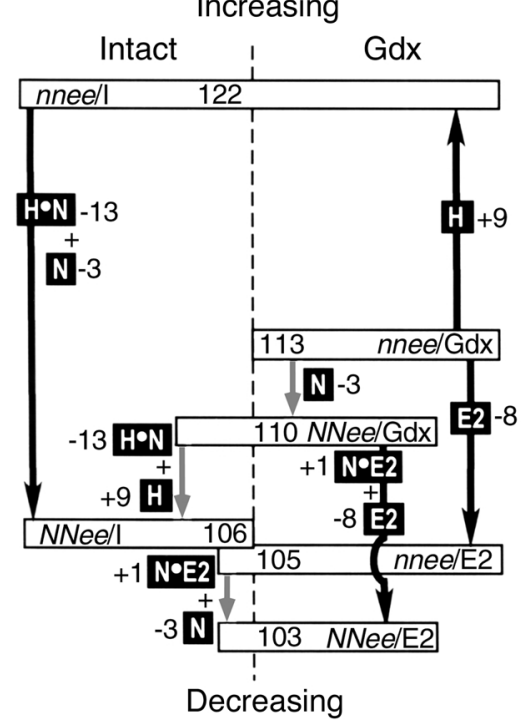

Figure 4

Path diagram: contribution of sex hormones, eNOS, and their interactions to BP (left panel) and atherosclerotic lesion size (right panel) in Apoe $e^{-/-}$mice. Average BP (in mmHg) and plaque size (in $\mu \mathrm{m}^{2} \times 10^{3}$ ) of the six groups of animals are indicated in rectangles and placed on the vertical scale. Black boxes indicate the presence of different components. $\mathrm{H}$ indicates hormone effects in the absence of eNOS, $\mathrm{N}$ indicates eNOS effects in the absence of sex hormones, E2 indicates exogenous E2 effects in the absence of eNOS and endogenous sex hormones, $\mathrm{H} \bullet \mathrm{N}$ indicates interaction between endogenous sex hormones and eNOS, and N•E2 indicates the interaction between eNOS and exogenous E2. Black arrows indicate statistically significant differences, and gray arrows indicate nonsignificant differences. Numbers next to black boxes represent the contribution of each effect on increasing or decreasing BP and lesion size. 
$\mathrm{H} \bullet \mathrm{N}$ interaction $\left(-71 \times 10^{3} \mu \mathrm{m}^{2}\right)$ and by E2 $\left(-56 \times 10^{3}\right.$ $\mu \mathrm{m}^{2}$ ). The effect of the $\mathrm{H} \bullet \mathrm{N}$ interaction is the largest on both BP and atherosclerosis. Minor differences between the effects on atherosclerosis and the effects on BP include a small atheropromotive contribution of eNOS in the absence of sex hormones, which in turn results in a small atheroprotective effect by the $\mathrm{H} \bullet \mathrm{E} 2$ interaction. However, neither of these effects is statistically significant. Note that the numbers assigned to each component in the two diagrams should not be taken too literally since they are based on the assumption that the effects on average BP or mean plaque size are algebraically additive. Also note that much of the change in $\mathrm{BP}$ and atherosclerosis that we observed was modest. The comparisons between intact and gonadectomized animals, in particular, should be repeated in parallel and with a larger number of animals to be confident of the magnitude of the effects. Nevertheless, although the experimental measurements of the two phenotypes are completely independent, the left and right sides of the diagram are remarkably similar and support the significance of $\mathrm{H}, \mathrm{H} \bullet \mathrm{N}$, and E2. It also illustrates that the two phenotypes, hypertension and atherosclerosis, are strongly associated.

Considerable evidence from both human and animal studies suggests that hypertension contributes to the progression of atherosclerosis (36). However, although experimentally reducing BP leads to a reduction in lesion size in nnee mice (22), the modest lowering of BP by the amount seen in NNee and nnee mice treated with E2 cannot account for the profound reduction in their lesion size. Similarly, endothelial dysfunction associated with atherosclerosis could raise BP. However, Apoe $\mathrm{Al}^{--}$ mice, even at 13 months of age when their lesions are large, have $\mathrm{BP}$ values that are no different from those measured in $\mathrm{Apoe}^{+/+}$mice of the same age $(22,28,37)$, indicating that lesion-induced endothelial dysfunction is unlikely to explain the BP changes in 4-month-old $\mathrm{Apoe}^{-/-}$mice. In our study, BP reduction by exogenous E2 is consistently less pronounced than reduction in lesion size. Thus the large atheroprotective effect of E2 we observed in our study is likely a result of the compound actions of exogenous E2, including lowering of BP and lipids, antioxidant properties, and inflammatory and immunoregulatory functions.

The path diagram presented in Figure 4 helps to conceptualize the relative contributions of each component in determining the two complex phenotypes. They show that the contribution of eNOS in basal BP regulation and in atheroprotective effects is largely through interactions with gonadal sex hormones. They also show that the endogenous sex hormone(s) whose interaction with eNOS $(\mathrm{H} \bullet \mathrm{N})$ contributes in a protective manner, and the endogenous sex hormone(s) that act in a damaging manner when eNOS is absent $(\mathrm{H})$, are present in both males and females. Current knowledge is insufficient to suggest the identity of the hormone or hormones responsible for these effects and how they interact with eNOS. Nevertheless, it is important to note that estrogen and androgen receptors are expressed at much lower levels in vascular tissues than in reproductive organs, and the ligand specificity of the receptors in nonreproductive tissues appears to be less stringent (38).

Particularly difficult to explain is the potential damaging effect of sex hormones in the absence of eNOS. The strong protective effect of exogenous E2 implies that it is gonad-derived hormone(s) other than E2 that exert these damaging effects. However, evidence exists that actions of sex hormones are biphasic and concentration-dependent. For example, experiments by Somjen et al. (39) have shown that low concentrations of both $\mathrm{E} 2$ and dihydrotestosterone have a proliferative effect on VSMCs in vitro (atheropromotive), but that high concentrations of these hormones inhibited proliferation (atheroprotective). Thus the effects of endogenous sex hormones in vivo, in the absence of eNOS, may well be a concentration-dependent phenomenon.

In conclusion, our studies have revealed a complex pattern of interactions between eNOS and sex hormones in vascular protection: (a) Endogenous sex hormones in the absence of eNOS have significant vascular damaging effects. (b) eNOS in the absence of endogenous sex hormones has little effect on the vasculature; yet (c) the interaction of eNOS and endogenous sex hormones results in a large protective effect. (d) Exogenous E2, when given continuously, clearly has powerful vasculoprotective action without eNOS. The effects of sex hormones on the cardiovascular system are concentration-dependent, and the balance between various hormones is likely to be very important. Much remains to be determined about the underlying mechanisms. Nevertheless, the current views, that premenopausal women are protected because of endogenous E2 and that E2 is vasculoprotective largely through its stimulative effects on NO formation and release, are overly simplistic. Only when specific components are individually dissected, as is possible in genetically altered mice, can the contributions of each component be separated for study. Our system provides a means to examine the contribution of each sex hormone, and its interaction with eNOS, to its vasculoprotective action.

\section{Acknowledgments}

The authors would like to thank Cam Patterson and CoraJean Edgell for helpful comments, and Shinja Kim and Virginia Godfrey for technical assistance. This work was supported by NIH grants HL-42630 (N. Maeda), HL-62845 (N. Maeda), HL-49277 (O. Smithies), and GM-20069 (O. Smithies).

\footnotetext{
1. Reckelhoff, J.F. 2001. Gender differences in the regulation of blood pressure. Hypertension. 37:1199-1208.

2. Chae, C.U., Ridker, P.M., and Manson, J.E. 1997. Postmenopausal hormone replacement therapy and cardiovascular disease. Thromb. Haemost. 78:770-780.

3. Mosca, L., et al. 2001. Hormone replacement therapy and cardiovascular disease: a statement for healthcare professionals from the American Heart Association. Circulation. 104:499-503.

4. Sullivan, M.L., Martinez, C.M.,Gennis, P., and Gallagher, E.J. 1998. The cardiac toxicity of anabolic steroids. Prog. Cardiovasc. Dis. 41:1-15.
} 
5. English, K.M., et al. 2000. Men with coronary artery disease have lower levels of androgens than men with normal coronary angiograms. Eur. Heart J. 21:890-894.

6. Bourassa, P.A., Milos, P.M., Gaynor, B.J., Breslow, J.L., and Aiello, R.J. 1996. Estrogen reduces atherosclerotic lesion development in apolipoprotein E-deficient mice. Proc. Natl. Acad. Sci. USA. 93:10022-10027.

7. Hough, J.L., and Zilversmit, D.B. 1986. Effect of 17 beta estradiol on aortic cholesterol content and metabolism in cholesterol-fed rabbits. Arteriosclerosis. 6:57-63.

8. Wagner, J.D., et al. 1991. Estrogen and progesterone replacement therapy reduces low density lipoprotein accumulation in the coronary arteries of surgically postmenopausal cynomolgus monkeys. J. Clin. Invest. 88:1995-2002.

9. Elhage, R., et al. 1997. 17 beta-estradiol prevents fatty streak formation in apolipoprotein E-deficient mice. Arterioscler. Thromb. Vasc. Biol. 17:2679-2684

10. Sasaki, T., et al. 2000. Oestrogen attenuates the increases in blood pressure and platelet aggregation in ovariectomized and salt-loaded Dahl salt-sensitive rats. J. Hypertens. 18:911-917.

11. Alexandersen, P., Haarbo, J., Byrjalsen, I., Lawaetz, H., and Christiansen, C. 1999. Natural androgens inhibit male atherosclerosis: a study in castrated, cholesterol-fed rabbits. Circ. Res. 84:813-819.

12. Nathan, L., et al. 2001. Testosterone inhibits early atherogenesis by conversion to estradiol: critical role of aromatase. Proc. Natl. Acad. Sci. USA 98:3589-3593

13. Grady, D., et al. 1992. Hormone therapy to prevent disease and prolong life in postmenopausal women. Ann. Intern. Med. 117:1016-1037.

14. Mendelsohn, M.E., and Karas, R.H. 1999. The protective effects of estrogen on the cardiovascular system. N. Engl. J. Med. 340:1801-1811.

15. Nathan, L., and Chaudhuri, G. 1997. Estrogens and atherosclerosis. Annu. Rev. Pharmacol. Toxicol. 37:477-515.

16. Akkad, A.A., Halligan, A.W., Abrams, K., and al-Azzawi, F. 1997. Differing responses in blood pressure over 24 hours in normotensive women receiving oral or transdermal estrogen replacement therapy. Obstet. Gynecol. 89:97-103.

17. Seely, E.W., Walsh, B.W., Gerhard, M.D., and Williams, G.H. 1999. Estradiol with or without progesterone and ambulatory blood pressure in postmenopausal women. Hypertension. 33:1190-1194.

18. Huang, P.L., et al. 1995. Hypertension in mice lacking the gene for endothelial nitric oxide synthase. Nature. 377:239-242.

19. Shesely, E.G., et al. 1996. Elevated blood pressures in mice lacking endothelial nitric oxide synthase. Proc. Natl. Acad. Sci. USA. 93:13176-13181.

20. Faraci, F.M., Sigmund, C.D., Shesely, E.G., Maeda, N., and Heistad, D.D. 1998. Responses of carotid artery in mice deficient in expression of the gene for endothelial NO synthase. Am. J. Physiol. 274:H564-H570.

21. Rudic, R.D., et al. 1998. Direct evidence for the importance of endothelium-derived nitric oxide in vascular remodeling. J. Clin. Invest. 101:731-736.
22. Knowles, J.W., et al. 2000. Enhanced atherosclerosis and kidney dys function in $\mathrm{eNOS}^{-/-} \mathrm{Apoe}^{-/-}$mice are ameliorated by enalapril treatment. J. Clin. Invest. 105:451-458.

23. Williams, J.K., Adams, M.R., and Klopfenstein, H.S. 1990. Estrogen modulates responses of atherosclerotic coronary arteries. Circulation. 81:1680-1687.

24. Weiner, C.P., et al. 1994. Induction of calcium-dependent nitric oxide synthases by sex hormones. Proc. Natl. Acad. Sci. USA. 91:5212-5216.

25. Holm, P., et al. 1997. Significant reduction of the antiatherogenic effect of estrogen by long-term inhibition of nitric oxide synthesis in cholesterol-clamped rabbits. J. Clin. Invest. 100:821-828.

26. Elhage, R., et al. 1997. Prevention of fatty streak formation of 17 betaestradiol is not mediated by the production of nitric oxide in apolipoprotein E-deficient mice. Circulation. 96:3048-3052.

27. Zhang, S.H., Reddick, R.L., Burkey, B., and Maeda, N. 1994. Diet-induced atherosclerosis in mice heterozygous and homozygous for apolipoprotein E gene disruption. J. Clin. Invest. 94:937-945.

28. Krege, J.H., Hodgin, J.B., Hagaman, J.R., and Smithies, O. 1995. A noninvasive computerized tail-cuff system for measuring blood pressure in mice. Hypertension. 25:1111-1115.

29. Knowles, J.W., et al. 2001. Pressure-independent enhancement of cardiac hypertrophy in natriuretic peptide receptor A-deficient mice. J. Clin. Invest. 107:975-984.

30. Kuhlencordt, P.J., et al. 2001. Accelerated atherosclerosis, aortic aneurysm formation, and ischemic heart disease in apolipoprotein E/endothelial nitric oxide synthase double-knockout mice. Circulation. 104:448-454

31. Hodgin, J.B., et al. 2001. Estrogen receptor $\alpha$ is a major mediator of $17 \beta$ estradiol's atheroprotective effects on lesion size in Apoe $e^{-/-}$mice. J. Clin. Invest. 107:333-340.

32. Krege, J.H., et al. 1997. Angiotensin-converting enzyme gene and atherosclerosis. Arterioscler. Thromb. Vasc. Biol. 17:1245-1250.

33. Bausero, P., Ben-Mahdi, M., Mazucatelli, J., Bloy, C., and PerrotApplanat, M. 2000. Vascular endothelial growth factor is modulated in vascular muscle cells by estradiol, tamoxifen, and hypoxia. Am. J. Physiol. Heart Circ. Physiol. 279:H2033-H2042.

34. Celletti, F.L., et al. 2001. Vascular endothelial growth factor enhances atherosclerotic plaque progression. Nat. Med. 7:425-429.

35. Kuroski de Bold, M.L. 1999. Estrogen, natriuretic peptides and the reninangiotensin system. Cardiovasc. Res. 41:524-531.

36. Knowles, J.W., and Maeda, N. 2000. Genetic modifiers of atherosclerosis in mice. Arterioscler. Thromb. Vasc. Biol. 20:2336-2345.

37. Hartley, C.J., et al. 2000. Hemodynamic changes in apolipoprotein Eknockout mice. Am. J. Physiol. Heart Circ. Physiol. 279:H2326-H2334.

38. Manolagas, S.C., and Kousteni, S. 2001. Perspective: nonreproductive sites of action of reproductive hormones. Endocrinology. 142:2200-2204

39. Somjen, D., et al. 1998. Effects of gonadal steroids and their antagonists on DNA synthesis in human vascular cells. Hypertension. 32:39-45. 